\title{
Importance of follow-up inspection after pulmonary angioplastic procedures for lung cancer surgery
}

\author{
Takeshi Nagayasu, MD, PhD
}

Received: 21 June 2009

(C) The Japanese Association for Thoracic Surgery 2010

In cases of lung cancer invading the pulmonary artery (PA), PA reconstruction with or without bronchoplasty has several advantages over pneumonectomy, including preservation of lung function and lower morbidity and mortality. I read with interest the article by Yamashita and colleagues ${ }^{1}$ who performed PA reconstruction for lung cancer surgery in 25 cases. As the authors suggest, I agree that pulmonary angioplasty procedure is a safe, acceptable alternative to pneumonectomy in lung cancer patients. Therefore, detailed study of the techniques and the indications for each case are indispensable for the surgeon. However, there are some aspects of the results that raise some issues and require further consideration.

First, the authors noted that the reason for performing PA plasty in all 25 cases was related to invasion from the primary tumors (14 cases) or lymph nodes (11 cases). However, they performed PA plasty with bronchoplasty (double plasty) in only six cases. During lung cancer surgery, we sometimes must determine how to manage pulmonary angioplastic procedures in cases with tumor or bulky hilar lymph node invasion. Pulmonary angioplasty without bronchoplasty is performed less often than double plasty because the lung cancer occurs from the lung parenchyma or bronchial wall. Rendina et al. reported 52 PA reconstructions for lung cancer associ-

This editorial refers to the article by Yamashita et al. on pp. 19-24 of this issue of General Thoracic and Cardiovascular Surgery.

T. Nagayasu $(\triangle)$

Division of Surgical Oncology, Department of Surgery,

Nagasaki University Graduate School of Biomedical Sciences,

1-7-1 Sakamoto, Nagasaki 852-8501, Japan

Tel. +81-95-819-7304; Fax +81-95-819-7306

e-mail: nagayasu@nagasaki-u.ac.jp ated with 33 bronchial sleeve resections; and PA plasty alone was indicated in less than $30 \%$ of all PA reconstruction $^{\text {cases. }}{ }^{2}$ In our institution over the past two decades, PA plasty alone was performed in $31 \%$ of all $\mathrm{PA}$ reconstructions. Therefore, the question remains as to the indication for PA plasty alone in this article. Although it is sometimes difficult to distinguish cancerrelated invasion from benign adhesions during surgery, we often treat patients with fibrous adhesions after chemoradiotherapy or calcified hilar lymph nodes with obstructive lung disease, which poses some difficulty in direct dissection of the PA segmental branch. In most of these cases, we do not chose circumferential or wedge PA resection but use small, tangential resection and sideto-side sutures. Of course, such cases should not be called PA plasty for lung cancer as there is no cancerrelated invasion.

Second, the authors preferred the technique of deep wedge resection of the PA and a proximal-end-to-distalend anastomosis to avoid stenotic change after primary side-to-side suturing of the PA. With double plasty, bronchial reconstruction brings the anastomotic end of the pulmonary artery closer and avoids kinking or angulation after end-to-end anastomosis. However, in cases of PA plasty alone, end-to-end anastomosis for wedge resection is sometimes not feasible because of the lack of this shortening effect of double plasty. The anastomotic shape after reconstruction of PA wedge plasty often proves to be disappointing. The authors reported that reconstruction of the length of the wedge-resected part of the PA within $3 \mathrm{~cm}$ did not cause any kinking or stricture. However, I believe that the way to avoid kinking after wedge resection of the PA is to cut the tangential direction as short as possible and cut the vertical direction as deep as possible. For cases of extended longitu- 
dinal defects in which end-to-end anastomosis is not feasible, I encourage the use of patch reconstruction.

Third, the authors did not routinely conduct followup inspections of the PA and bronchial anastomosis. I would emphasize that follow-up inspection of the anastomosis after PA or bronchial reconstruction is essential for the surgeon to improve and provide feedback related to surgical techniques. Instead of conventional PA angiograms, recent advances in multirow detector computed tomography (MDCT) enable clear three-dimensional visualization of even small kinking or angulation of the anastomosis site without invasiveness. For bronchial anastomosis, I routinely use a conventional flexible bronchoscope to inspect the site within 2 weeks after operation, although virtual endoscopy may take the place of conventional flexible bronchoscopy in the near future.
Finally, I believe that pulmonary angioplastic procedures for lung cancer have expanded the surgical indications for marginal patients with low pulmonary function and improved patients' quality of life by avoiding pneumonectomy. Therefore, it is important to study this special technique continuously while sharing information.

\section{References}

1. Yamashita M, Komori E, Sawada S, Suehisa H, Nozaki I, Kurita A, et al. Pulmonary angioplastic procedure for lung cancer surgery. Gen Thorac Cardiovasc Surg 2010;58:19-24.

2. Rendina EA, Venuta F, De Giacomo T, Ciccone AM, Moretti $\mathrm{M}$, Ruvolo $\mathrm{G}$, et al. Sleeve resection and prosthetic reconstruction of the pulmonary artery for lung cancer. Ann Thorac Surg 1999;68:995-1001; discussion 1001-2. 